\title{
Nonlinear noninertial response of a quantum Brownian particle in a tilted periodic potential to a strong ac force as applied to a point Josephson junction
}

\author{
William T. Coffey, ${ }^{1}$ Yuri P. Kalmykov, ${ }^{2}$ Serguey V. Titov, ${ }^{3}$ and Liam Cleary ${ }^{1}$ \\ ${ }^{1}$ Department of Electronic and Electrical Engineering, Trinity College, Dublin 2, Ireland \\ ${ }^{2}$ Laboratoire de Mathématiques, Physique et Systèmes, Université de Perpignan, 52, Avenue de Paul Alduy, \\ 66860 Perpignan Cedex, France \\ ${ }^{3}$ Kotel'nikov Institute of Radioengineering and Electronics, Russian Academy of Sciences, Vvedenskii Square 1, \\ Fryazino, 141190, Russian Federation \\ (Received 4 November 2008; published 10 February 2009)
}

\begin{abstract}
The quantum Smoluchowski equation for the reduced Wigner function in configuration space pertaining to the quantum Brownian motion of a particle in a tilted cosine potential in the high dissipation (or noninertial) limit as applied to a model point Josephson junction (namely, a resistively shunted junction in the presence of noise and an arbitrarily large microwave ac driving current) is considered. The solution of the resulting recurrence relations for the Fourier amplitudes of the statistical moments describing the nonlinear dynamics of the junction (ignoring the capacitance) is obtained using the matrix continued fractions previously developed for the stationary ac field solution of the corresponding classical problem. Quantum effects in the nonlinear response of the junction to an ac microwave current of arbitrary amplitude (nonlinear microwave impedance, frequency dependence of the dc current-voltage characteristic, etc.) are estimated.
\end{abstract}

DOI: 10.1103/PhysRevB.79.054507

PACS number(s): 74.50. $+\mathrm{r}, 05.40 .-\mathrm{a}, 03.65 . \mathrm{Yz}$

\section{INTRODUCTION}

Wigner's phase-space formulation of quantum mechanics in terms of quasiprobability distributions of the canonical variables $^{1-7}$ as extended to open quantum systems (see, e.g., Refs. 8-16) has recently been used ${ }^{15,17}$ to derive a quantum Smoluchowski equation (QSE) governing the time evolution of the configuration-space distribution function $P(x, t)$ for particles with separable and additive Hamiltonians in the overdamped (or noninertial) limit. In the present context, pertaining to a quantum Brownian particle of mass $m$ moving along the $x$ axis under the influence of a potential $V(x)$, the canonical variables are the position $x$ and the momentum $p$ of the particle. The corresponding reduced (single-particle) joint quasiprobability distribution function in phase space, namely, the Wigner function $W(x, p, t)$, represents the projection of all the other degrees of freedom of the system, comprised of the quantum Brownian particle and its heat bath, onto the phase space $(x, p)$ of that particle. The evolution of $W(x, p, t)$ is governed by the master equation ${ }^{8}$

$$
\begin{aligned}
& \frac{\partial W}{\partial t}+\frac{p}{m} \frac{\partial W}{\partial x}-\frac{1}{i \hbar}\left[V\left(x+\frac{i \hbar}{2} \frac{\partial}{\partial p}\right)-V\left(x-\frac{i \hbar}{2} \frac{\partial}{\partial p}\right)\right] W \\
& =\hat{M}_{D} W
\end{aligned}
$$

where $\hat{M}_{D}$ is the collision kernel operator representing the bath-particle interaction and $\hbar$ is Planck's constant. Here the left-hand side is the single-particle Wigner equation ${ }^{1,6,8}$ (which is the quantum analog of the classical Liouville equation) governing the evolution of the joint quasiprobability distribution function for the closed system. The stationary solution of this equation [i.e., the master Eq. (1) with the right-hand side equal to zero] is the Wigner stationary distribution $W_{0}(x, p)$, which can be developed as a power series in $\hbar^{2}$, viz., ${ }^{1}$

$$
\begin{aligned}
W_{0}(x, p)= & e^{-\beta \varepsilon(x, p)}\left\{1+\frac{(\beta \hbar)^{2}}{24 m}\left[\beta V^{\prime 2}(x)-\left(3-\frac{\beta p^{2}}{m}\right) V^{\prime \prime}(x)\right]\right. \\
& +\cdots\},
\end{aligned}
$$

where $\varepsilon(x, p)=p^{2} /(2 m)+V(x)$ is the classical energy of the particle, $\beta=(k T)^{-1}, k$ is Boltzmann's constant, and $T$ is the temperature. Regarding the general open system governed by Eq. (1), various forms of the collision operator $\hat{M}_{D}$ have been discussed in detail in Ref. 8. Now, on specializing to the quantum Brownian motion in the high-temperature and weak-coupling limits, the collision operator $\hat{M}_{D}$ can be represented just as in the classical theory by a Kramers-Moyal expansion truncated at the second term. However, unlike the classical theory in order that $W_{0}(x, p)$ should also render the right-hand side of Eq. (1) zero, the coefficients of the truncated Kramers-Moyal expansion must become functions of the derivatives of the potential. The master Eq. (1) in phase space then describes the relaxation of $W(x, p, t)$ to the stationary state given by $W_{0}(x, p)$ in the long time limit. ${ }^{15,17}$ The ansatz that the Wigner stationary distribution $W_{0}(x, p)$ renders the right-hand side of the master Eq. (1) zero (whence the diffusion coefficients must depend on the derivatives of the potential) may be used if the interactions between the Brownian particle and the heat bath are small enough to allow one to use the weak-coupling limit, and if the correlation time characterizing the bath is so short that one can regard the stochastic process originating in the bath as Markovian. For parameter ranges, where such an approximation is invalid (e.g., throughout the very-low-temperature region, where non-Markovian effects are substantial), other methods should be used. ${ }^{9}$ We remark that the imposition of $W_{0}(x, p)$ as the stationary solution of Eq. (1) is exactly analogous to the assumption of the Maxwell-Boltzmann distribution as the 
stationary distribution in the classical Brownian motion. ${ }^{18}$ The configuration-space distribution $P(x, t)$ may then be calculated by integration of $W(x, p, t)$ over the momenta, i.e.,

$$
P(x, t)=\int W(x, p, t) d p .
$$

Now, in general ${ }^{18}$ in both the classical and quantum cases it is impossible to write a partial differential equation in configuration space describing the evolution of the probability distribution $P(x, t)$. The sole exception, however, is the overdamped (or noninertial) limit, where the governing equation is the quantum Smoluchowski equation ${ }^{15,17}$

$$
\frac{\partial P(x, t)}{\partial t}=\frac{\partial}{\partial x}\left\{\frac{P(x, t)}{\zeta} \frac{\partial V(x)}{\partial x}+\frac{\partial}{\partial x}[D(x) P(x, t)]\right\} .
$$

Here $D(x)$ is the quantum diffusion coefficient which depends on the derivatives of the potential and which is represented as a power series in $\hbar^{2},{ }^{15,17} \zeta=\gamma m$ is the friction coefficient, $\gamma$ is a dissipation (damping) parameter characterizing the bath-particle interaction, and the drift coefficient $-\zeta^{-1} \partial_{x} V$ coincides with its classical counterpart. The quasiprobability density $P(x, t)$ is simply the trace of the densitymatrix operator $\hat{\rho} .^{4-6}$

Here we apply the QSE (3) to evaluate quantum corrections to the nonlinear noninertial response to a strong ac force $f \cos \omega t$ of a Brownian particle moving in a tilted cosine (or inclined washboard) potential with amplitude $V_{0}$, characteristic length $a$, and constant tilt (slope) $F$, viz.,

$$
V(x)=-V_{0} \cos (2 \pi x / a)-x F-x f \cos \omega t .
$$

We first remark that the overdamped Brownian motion of a particle in a tilted cosine potential arises in a number of important physical applications. We mention Josephson junctions, ${ }^{19-21}$ superionic conductors, ${ }^{22}$ ring-laser gyroscopes, ${ }^{23}$ phase-locking techniques in radio engineering, ${ }^{24}$ charged density waves,${ }^{25}$ diffusion of colloidal particles in periodic structures, ${ }^{26}$ dynamics of vortices in superconductors, ${ }^{27}$ etc. As a particular example, we shall estimate quantum effects on the nonlinear dynamics of a point Josephson junction in the zero-capacitance limit (so-called RSJ model) ${ }^{19-21}$ initiated by Ambegaokar and Halperin ${ }^{28}$ and by Ivanchenko and Zil'berman. ${ }^{29}$ Despite its limitations, ${ }^{19-21}$ the RSJ model yields for the linear response of the junction to an ac driving current a relatively simple treatment of both the dc current-voltage characteristic and the impedance both in the classical ${ }^{19-21,30}$ and quantum ${ }^{31}$ cases. In the quantum case, the dc current-voltage characteristic and the differential resistance may be expressed ${ }^{31}$ as modified Bessel functions of the first kind just as in the classical limit. ${ }^{30}$ Moreover, the linear impedance characteristic resembles that of a simple resonant circuit, where the real part exhibits a pronounced minimum at the resonant frequency and the imaginary part a pronounced maximum. In particular, the quantum effects are discernible in the linear response as an enhanced current for a given voltage in the dc current-voltage characteristic and an enhanced slope in the differential-resistance, which, besides the impedance, are the other quantities of physical interest. ${ }^{31}$ As far as the dc characteristics in the linear regime are concerned, the quantum effects $\operatorname{arise}^{31}$ due to hightemperature nondissipative tunneling near the top of a barrier and are readily detectable for a relatively large supercurrent and small bias. This behavior is due to the effective reduction in the barrier height, associated with a well of the inclined washboard potential. This barrier lowering mechanism due to high-temperature quantum tunneling was originally identified by Wigner in his quantum transition state theory, ${ }^{1,32}$ which of course ignores the dissipation to the bath. On the other hand, in the resonant peak in the impedance curve, the quantum effects manifest themselves essentially ${ }^{31}$ as an enhancement of the $Q$ factor, which is an example of dissipative tunneling reducing the damping of the Josephson oscillations.

However, the restriction of the treatment of the Josephson junction to linear response means that many important features of both the dc and the ac microwave behavior cannot be reproduced. For example, in the $d c$ response these include the ubiquitous Shapiro $\operatorname{steps}^{33}$ in the obviously timeindependent (but frequency-dependent) dc current-voltage characteristics. The steps are due to dynamical phase slips caused by change in the phase locking of the Josephson oscillator at multiple harmonics of the frequency of the applied ac current as that current is increased. This behavior constitutes a form of modulation due to nonlinear effects. In the ac response, other nonreproducible effects include the manifold oscillations (see, e.g., Refs. 34-36 and references cited therein) appearing in the reactive part of the nonlinear impedance (again due to dynamical phase slips) and the threshold points and saturation behavior of the resistive part, whereby the dynamic resistance approaches the shunt resistance of the junction. Hitherto, the nonlinear ac response (mainly, the microwave resistance and reactance) to a strong probing ac current has usually been calculated via perturbation theory (e.g., Ref. 34). However, this approach is valid for small ac current amplitudes only, or in the noiseless limit, where the governing nonlinear equation of motion can be solved numerically (e.g., Refs. 35 and 37). As far as experimental observations are concerned, nonlinear effects in the microwave resistance have been observed and explained (using the classical RSJ model) in Refs. 36 and 37. Experimental data ${ }^{38}$ on the nonlinear microwave surface impedance $Z_{s}$ of high-temperature superconducting thin films have also been interpreted ${ }^{39-41}$ using the classical RSJ model, and qualitative agreement with experimental measurements of $Z_{s}$ has been reported, showing that these systems also behave like a Josephson junction. Moreover, the RSJ model can also describe the main features of the nonlinear surface impedance, namely, the steps in the ac current dependence of the resistance, ${ }^{33,34}$ the threshold points, ${ }^{40,41}$ and saturation. ${ }^{40,41}$ Among other experiments, which can be analyzed using the classical RSJ model, are microwave absorption measurements in weak-link Josephson junctions ${ }^{42}$ in high- $T_{c}$ superconductors, where the microwave surface resistance $R_{s}$ was studied. Furthermore, as shown in Ref. 42, the numerical solution of the nonlinear dynamical equation governing the noiseless RSJ model agrees closely with experiment. Finally, the nonlinear ac response of a point Josephson junction in the presence of noise has been evaluated in Refs. 43 and 44 using the matrix continued fraction technique. This technique 
has also been $u \mathrm{~d}^{27}$ for the solution of a very similar Langevin equation for a two-dimensional nonlinear guided vortex motion in a tilted cosine pinning potential in the presence of an ac for arbitrary values of the Hall effect.

Mindful of the several distinctive features of the nonlinear stationary response, which we have summarized above, it is the purpose of this paper to extend the calculations of the classical nonlinear stationary response $\mathrm{s}^{43,44}$ to the quantum nonlinear stationary response in the zero-capacitance limit. This will be accomplished by solving the QSE (3) for the nonlinear stationary ac response in a tilted cosine potential for an ac driving current of arbitrary amplitude using the matrix continued fraction method as developed in Refs. 43 and 44 for the corresponding classical problem. In particular, we shall calculate the nonlinear impedance and the frequency-dependent dc current-voltage characteristics, noting that certain aspects of quantum effects in the characteristics of Josephson junctions have already been analyzed, e.g., in Refs. 45-49. We remark that a matrix-continued fraction is invariably involved in the solution of the nonlinear response rather than the scalar continued fraction of the linear response. This is so because the distribution function for the phase variable must now be expanded in a double Fourier series in both space and time because of the coupling between the time-varying harmonic components of the driving force induced by the nonlinearity. This behavior is of course not evident in the linear-response approximation where the ac response is simply the stimulus shifted in amplitude and phase (so that no harmonics of the stimulus occur) along with a dc response independent of the ac response.

\section{QSE FOR THE RSJ MODEL}

The Josephson junction as described by the RSJ model comprises two superconductors separated by a thin layer of oxide. The phase difference $\varphi=\varphi_{l}-\varphi_{r}$ between the wave functions of the right and left superconductors is given by the Josephson equation ${ }^{19-21}$

$$
\frac{d}{d t} \varphi(t)=\frac{2 e v(t)}{\hbar}
$$

where $v(t)$ is the potential difference across the oxide layer and $e$ is the charge of the electron. If the junction is small enough (a point Josephson contact), it may be modeled ${ }^{19-21}$ by a resistance $R$ in parallel with a phase-dependent current generator, $I \sin \varphi$, representing the Josephson supercurrent due to the Cooper pairs tunneling through the junction, which has capacitance $C$. The junction is connected to an external current generator $I_{\mathrm{ex}}$ (representing the bias current applied to the junction). We suppose that the current $I_{\mathrm{ex}}$ consists of a dc current $I_{\mathrm{dc}}$ and an ac current $I_{\mathrm{ac}}=I_{m} \cos \omega t$ of arbitrary amplitude $I_{m}$. In the classical RSJ model, the dynamics of the junction (in the noninertial limit) in the presence of thermal agitation are described by the classical Smoluchowski equation ${ }^{19-21,28,29}$ for the time evolution of the configuration-space distribution function. This equation is formally equivalent to that describing a Brownian particle moving in a tilted cosine potential in the high dissipation limit. Thus, the junction is now treated as a purely classical system, where the phase difference $\varphi$ across the junction and the charge $v C$ on the junction are considered as classical variables which can be determined with arbitrary accuracy. ${ }^{21}$ However, the classical accuracy is inherently limited by Heisenberg's uncertainty principle, which in this case is $\Delta \varphi \Delta N \geq 1$, where $N$ is the number of Cooper pairs transferred across the junction. Hence, the results of classical theory (in particular, those arising from the classical Smoluchowski equation) require modification when quantum effects become important, e.g., at very low temperatures $T$ $<0.1 \mathrm{~K}^{21}$ Noting that the mass $m$ and the friction coefficient $\zeta$ of the mechanical Brownian particle are replaced in Eq. (3) by the corresponding electrical parameters $R$ and $C$ via $m=C(\hbar / 2 e)^{2}$ and $\zeta=(\hbar / 2 e)^{2} / R,{ }^{19-21,30,31}$ the QSE for the reduced Wigner function in configuration space $P(\varphi, t)$ is given by Eq. (3), viz.,

$$
\frac{\partial P(\varphi, t)}{\partial t}=\frac{\partial}{\partial \varphi}\left\{\frac{P(\varphi, t)}{\zeta} \frac{\partial U(\varphi)}{\partial \varphi}+\frac{\partial}{\partial \varphi}[D(\varphi) P(\varphi, t)]\right\},
$$

where the potential $U(\varphi)$ is

$$
U(\varphi)=-\beta^{-1} \gamma[\cos \varphi+\varphi(\alpha+\xi \cos \omega t)]
$$

$\gamma=\hbar I \beta /(2 e)$ is the normalized Josephson coupling energy (the parameter $\gamma$ also characterizes the noise strength), $\alpha$ $=I_{\mathrm{dc}} / I$ and $\xi=I_{m} / I$ are, respectively, the ratios of the dc and ac current amplitudes to the supercurrent amplitude (tilt and nonlinear parameters), and the diffusion coefficient $D$ is

$$
\begin{aligned}
D(\varphi)= & \frac{1}{\zeta \beta}\left\{1+\beta \Lambda U^{\prime \prime}(\varphi)-\frac{(\beta \Lambda)^{2}}{5}\left\{\left[U^{\prime \prime}(\varphi)\right]^{2}\right.\right. \\
& \left.\left.+3 U^{\prime}(\varphi) U^{(3)}(\varphi)-3 \beta^{-1} U^{(4)}(\varphi)\right\}+\ldots\right\} .
\end{aligned}
$$

Here $(\zeta \beta)^{-1}$ is the classical diffusion coefficient and $\Lambda$ $=e^{2} \beta /(3 C)$ is the dimensionless parameter corresponding to the quantum parameter $\hbar^{2} \beta /(12 m)$ for a mechanical Brownian particle. ${ }^{15}$ The QSE (6) is formally equivalent to the diffusion equation in configuration space describing classical noninertial Brownian motion in a potential with coordinatedependent diffusion coefficient $D$. Thus, the dynamics of the system described by the QSE (6) may be equivalently described using a quantum analog of the noninertial Langevin equation with multiplicative noise (see Appendix A). In the classical limit $\Lambda=0$, Eq. (6) reduces to the Smoluchowski equation used by Ambegaokar and Halperin ${ }^{28}$ and by IVanchenko and Zil'berman. ${ }^{29}$ The conditions for the validity of the QSE (6) are discussed in Ref. 31. We remark that the mathematical form of the leading quantum correction term in the diffusion coefficient $D(\varphi)$ is similar to that derived by entirely different reasoning in Refs. 8 and 49.

Now, quantum effects in the nonlinear (as well as in the linear) impedance of a point Josephson junction should be detectable when the quantum correction terms in Eq. (8) [e.g., $\beta \Lambda U^{\prime \prime}(\varphi)$, etc.] are comparable with unity, i.e., when the model parameter values are such that 


$$
\Lambda \gamma=\frac{\gamma \beta e^{2}}{3 C} \approx 0.6 \times 10^{-3} \frac{\gamma}{C[\mathrm{pF}] T[\mathrm{~K}]} \geq 0.1 \div 0.2
$$

(The parameter $\Lambda \gamma$ must not be too large in order to guarantee convergence of the perturbation expansion of $D$ in $\Lambda$ ). According to Eq. (9), the quantum effects should become more pronounced at lower temperatures $T$, smaller junction capacitances $C$, and larger dimensionless Josephson coupling energies $\gamma$. For example, with $\gamma \sim 15$ and $C \sim 1 \mathrm{pF}$, these effects are negligible in the temperature range $T>1 \mathrm{~K}$; however, they can play a vital role at temperatures below $0.1 \mathrm{~K}$. We remark that $\Lambda \gamma$ is very small for values of $T, \gamma$, and $C$ for typical Josephson junctions, as studied experimentally, for example, in Refs. 50-53 (here the current-voltage characteristics of Josephson junctions have been measured and compared with the RSJ model of Ambegaokar and Halperin ${ }^{28}$ and Ivanchenko and Zil'berman ${ }^{29}$ ). Thus, use of the classical RSJ model in these cases ${ }^{50-53}$ is entirely justified.

\section{NONLINEAR RESPONSE: MATRIX CONTINUED FRACTION SOLUTION}

In order to calculate the nonlinear impedance of the Josephson junction from the QSE (6), we note that the spatially periodic function $P(\varphi, t)$ can be expanded in a Fourier series in $\varphi,{ }^{18,30}$ viz.

$$
P(\varphi, t)=\frac{1}{2 \pi} \sum_{n=-\infty}^{\infty} c_{n}(t) e^{i n \varphi} .
$$

By substituting Eq. (10) into the QSE (6), we then find that the Fourier coefficients (statistical moments) $c_{n}(t)$ $=\left\langle e^{-i n \varphi}\right\rangle(t)$ satisfy the five-term differential recurrence relation to second order in the quantum correction parameter $\Lambda$

$$
\begin{aligned}
& \tau \frac{d}{d t} c_{n}(t)+\left[n^{2}+\frac{(\gamma n \Lambda)^{2}}{5}+i n \gamma(\alpha+\xi \cos \omega t)\right] c_{n}(t) \\
& \quad=\frac{\gamma n}{2}\left[(1-n \Lambda) c_{n-1}(t)-(1+n \Lambda) c_{n+1}(t)\right] \\
& \quad+\frac{\gamma n^{2} \Lambda^{2}}{10}\left\{2 \gamma c_{n-2}(t)+3[1-i \gamma(\alpha+\xi \cos \omega t)] c_{n-1}(t)\right. \\
& \left.+3[1+i \gamma(\alpha+\xi \cos \omega t)] c_{n+1}(t)+2 \gamma c_{n+2}(t)\right\}+O\left(\Lambda^{3}\right)
\end{aligned}
$$

Here

$$
\tau=\left(\frac{\hbar}{2 e}\right)^{2} \frac{\beta}{R}
$$

is the characteristic relaxation time. The recurrence relation, Eq. (11), may also be obtained directly from the appropriate quantum Langevin equation by averaging that equation over its realizations in configuration space as is illustrated in Appendix A.

Here, we are solely concerned with the nonlinear stationary ac response, where the ac has been applied for a very long time, i.e., we seek a solution independent of the initial conditions. Thus we make the following perturbation expansion:

$$
c_{n}(t)=\sum_{k=-\infty}^{\infty} c_{n}^{k}(\omega) e^{i k \omega t}
$$

accounting for the infinite number of harmonics of the stimulus produced by the nonlinear characteristics of the junction. The Fourier coefficients have the following properties $c_{n}^{k}(\omega)=c_{-n}^{k *}(-\omega)=c_{-n}^{-k *}(\omega)$ arising ultimately from the fact that $P(\varphi, t)$ must be real (asterisk denotes complex conjugate). By substituting Eq. (12) into Eq. (11), we have the dualindex algebraic recurrence relation for the Fourier amplitudes $c_{n}^{k}(\omega)$, viz.,

$$
\begin{aligned}
& i z_{n}^{k}(\omega) c_{n}^{k}(\omega)+q_{n}^{+} c_{n+1}^{k}(\omega)+q_{n}^{-} c_{n-1}^{k}(\omega)+\bar{q}_{n}\left[c_{n-2}^{k}(\omega)+c_{n+2}^{k}(\omega)\right] \\
& +i \xi\left\{c_{n}^{k-1}(\omega)+c_{n}^{k+1}(\omega)-(3 / 4) \bar{q}_{n}\left[c_{n-1}^{k-1}(\omega)+c_{n-1}^{k+1}(\omega)\right.\right. \\
& \left.\left.\quad-c_{n+1}^{k-1}(\omega)-c_{n+1}^{k+1}(\omega)\right]\right\}=0,
\end{aligned}
$$

where $n$ and $k$ are integers varying from $-\infty$ to $\infty, c_{0}^{0}(\omega)=1$, $c_{0}^{k}(\omega)=0(k>0)$, and

$$
\begin{gathered}
z_{n}^{k}(\omega)=2\left(\frac{k \omega \tau}{n \gamma}-\frac{i n}{\gamma}+\alpha-\frac{i \gamma n \Lambda^{2}}{5}\right), \\
q_{n}^{ \pm}= \pm\left[1 \pm n \Lambda \mp 3 n \Lambda^{2}(1 \pm i \gamma \alpha) / 5\right], \\
\bar{q}_{n}=-2 \gamma n \Lambda^{2} / 5 .
\end{gathered}
$$

Thus, invoking the familiar general matrix continued fraction method for solving multi-index recurrence relations generated by Fokker-Planck equations, ${ }^{18,30}$ one may obtain the solution of the differential recurrence Eq. (13) (details of this solution are given in Appendix B).

An intrinsic feature of the nonlinear response is the modulation of the dc current-voltage characteristic, giving rise, for example, to the famous Shapiro steps originating in the phase locking. Here, the time-independent but frequencydependent dimensionless average dc voltage $\langle\eta\rangle=\langle v\rangle / R I$ in the presence of an alternating force $\xi \cos \omega t$ is given by

$$
\langle\eta\rangle=\alpha+\operatorname{Im}\left[c_{1}^{0}(\omega)\right],
$$

while the nonlinear impedance is given by [recalling that $\left.c_{-1}^{k}(\omega)=c_{1}^{k *}(-\omega)\right]$

$$
R_{\omega}-i X_{\omega}=R\left\{1-i \xi^{-1}\left[c_{1}^{1}(\omega)-c_{1}^{1 *}(-\omega)\right]\right\},
$$

where $R_{\omega}$ and $X_{\omega}$ are the dynamic resistance and the reactance, respectively.

\section{RESULTS AND DISCUSSION}

In Fig. 1 we show the averaged dc voltage $\langle\eta\rangle$ versus the normalized dc bias current $\alpha$ for fixed frequency $\omega \tau$ and coupling energy $\gamma$, showing the stimulus-induced Shapiro steps occurring at integer multiples of the fundamental frequency due to dynamical phase slips and the difference between the quantum and classical results. In Fig. 2 we show the average frequency-dependent dc voltage $\langle\eta\rangle$ for various values of the applied ac current $\xi$. The effect of nonlinearity is to modulate the $\mathrm{dc}$ response, i.e., to induce large frequency-dependent troughs and peaks in the previously 


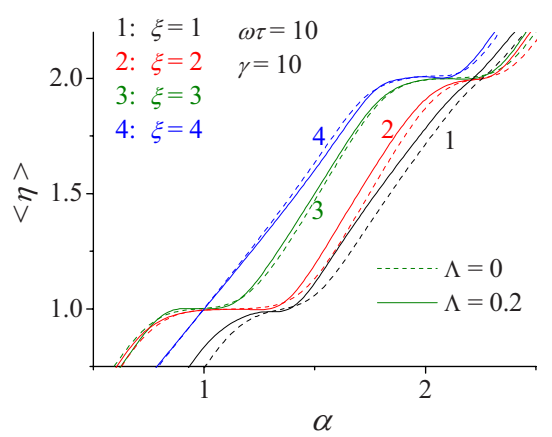

FIG. 1. (Color online) Averaged dc voltage $\langle\eta\rangle=\langle v\rangle /$ RI vs normalized dc bias current amplitude $\alpha=I_{\mathrm{dc}} / I$ for various values of the normalized ac amplitude $\xi=I_{m} / I$, fixed frequency $\omega \tau=10$, and coupling energy $\gamma=10$ showing the stimulus induced Shapiro steps for classical (dashed lines) and quantum (solid lines; $\Lambda=0.2$ ) cases.

constant curve of the dc voltage vs normalized frequency $\omega \tau$ characteristic of the linear response. In other words the timedependent response now affects the dc response. In general the effect of the quantum corrections for the ac amplitude between 0.1 and 1.0 is to enhance the troughs and peaks in the response. However, as the amplitude of the driving current increases above unity, the quantum effects decrease so that the quantum curves are very close to the corresponding classical curves, except at high frequencies, where the quantum peaks decrease more slowly. Thus it appears in this instance that relatively large nonlinear effects will mask the quantum effects. The enhancement of the nonlinear effects at intermediate amplitudes of the applied current is also evident in Fig. 3, where for all coupling energies the quantum effects enhance the relative heights of the classical peaks and troughs and also cause a shift of these extrema toward higher frequencies. This behavior appears to be entirely consistent with the enhancement of the $Q$ factor caused by dissipative quantum tunneling near the top of the barrier (reducing the damping of the Josephson oscillations) in the linear-response impedance characteristic ${ }^{31}$ and is an example of how dissi-

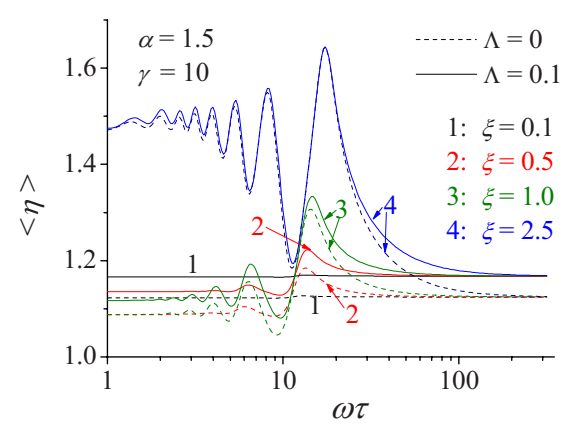

FIG. 2. (Color online) Averaged de voltage $\langle\eta\rangle$ vs dimensionless frequency $\omega \tau$ for various values of the applied ac stimulus $\xi$ for classical (dashed lines) and quantum (solid lines) cases. Strong nonlinearity, corresponding to large $\xi$, causes pronounced oscillatory behavior of the dc voltage with numerous frequency-dependent troughs and peaks as opposed to the smooth behavior associated with the linear response. The quantum effects, which are most pronounced at intermediate $\xi$ (curves 1,2 , and 3), comprising an increase in amplitude of the extrema and a shift of these to higher frequencies, diminish as $\xi$ increases (curve 4).

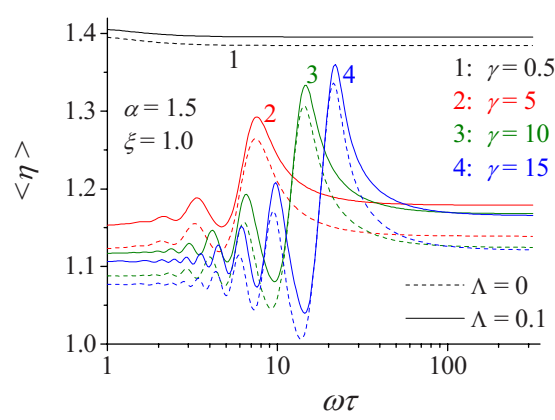

FIG. 3. (Color online) $\langle\eta\rangle$ vs $\omega \tau$ for various values of the normalized Josephson coupling energy $\gamma=\hbar I \beta /(2 e)$ (or noise strength) parameter and ac strength $\xi=1$; classical (dashed lines) and quantum (solid lines) results. The quantum effects enhance the nonlinear behavior for all values of $\gamma$.

pative tunneling can now affect the dc characteristics. The convergence of the perturbation procedure in $\Lambda$ is demonstrated in Fig. 4 by comparing the first and second order of perturbation-theory solutions. Here we show the averaged dc voltage vs frequency for various values of $\Lambda$ to first (dashed lines) and second (solid lines) order in $\Lambda$ showing that the quantum effects invariably enhance the troughs and peaks [the dotted line corresponds to the classical solution, i.e., the recurrence Eq. (11) ignoring all quantum terms]. Clearly, the first-order perturbation solution closely approximates the second-order one for small values of $\Lambda<0.2 / \gamma$. Moreover, inclusion of the second-order term is essential for the calculations at higher values of $\Lambda$ as is obvious in curve 3 . Another interesting point concerning Fig. 4 is that the main peak in the frequency curve is so enhanced in comparison to the classical case as to be experimentally detectable. Furthermore, its height is rather sensitive to $\Lambda$ as is again obvious from curve 3 of Fig. 4. We remark that the diffusion coefficient $D(\varphi)$ in Eq. (8) is written explicitly to $o\left(\Lambda^{2}\right)$ and that the greater the values of $\Lambda$ and $\gamma$, the higher the order of perturbation theory required. Higher-order correction terms to the diffusion coefficient $D(\varphi)$ may be calculated as described in Ref. 31. Hence, $D(\varphi)$ can be given, in principle, to any desired degree $r$ of $\Lambda^{r}$. For example, the next term in the perturbation expansion of $D(\varphi)$ in Eq. (8) is

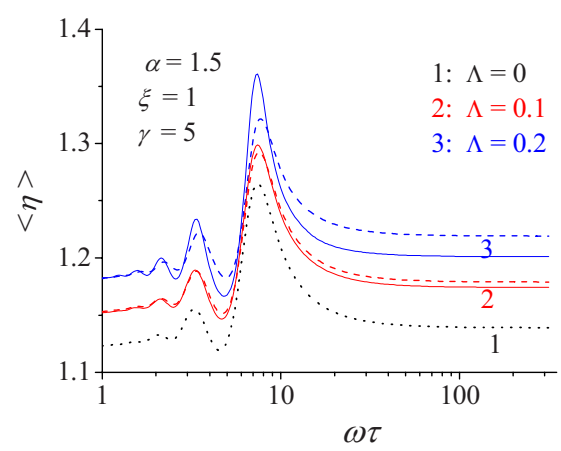

FIG. 4. (Color online) $\langle\eta\rangle$ vs $\omega \tau$ for various values of the quantum parameter $\Lambda$ to first- (dashed lines) and second- (solid lines) order quantum corrections in $\Lambda$, showing throughout that the quantum effects emphasize the troughs and peaks. Dotted line: classical limit, $\Lambda=0$. Inclusion of the second-order term is essential for higher values of $\Lambda=0.2$ (curve 3 ). 


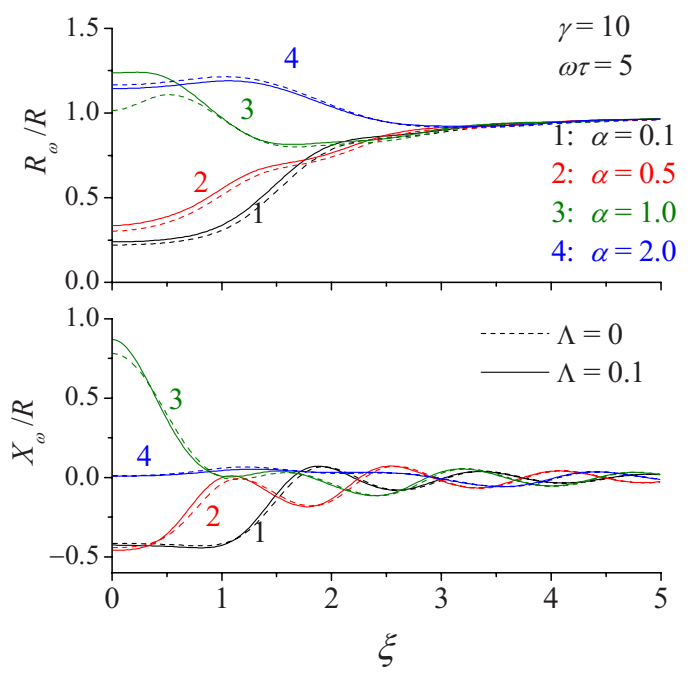

FIG. 5. (Color online) Normalized dynamic resistance $R_{\omega}$ and reactance $X_{\omega}$ vs applied ac stimulus amplitude $\xi$ for various dc bias current amplitudes $\alpha$ and $\gamma=10, \omega \tau=5$, showing strong nonlinearity for small bias and ohmic-resistance-like behavior for high bias. Dashed and solid lines are the classical and quantum results, respectively.

$$
\begin{aligned}
& +\frac{\beta^{2} \Lambda^{3}}{35 \zeta}\left\{2\left[U^{\prime \prime}(\varphi)\right]^{3}+12 U^{\prime \prime}(\varphi)\left[3 U^{(3)}(\varphi) U^{\prime}(\varphi)-2 \beta^{-1} U^{(4)}(\varphi)\right]\right. \\
& +9\left\{\left[U^{\prime}(\varphi)\right]^{2} U^{(4)}(\varphi)-2 \beta^{-1} U^{\prime}(\varphi) U^{(5)}(\varphi)+\beta^{-2} U^{(6)}(\varphi)\right\} \\
& \left.-30 \beta^{-1}\left[U^{(3)}(\varphi)\right]^{2}\right\} .
\end{aligned}
$$

In Fig. 5 we show the normalized dynamic resistance $R_{\omega}$ and reactance $X_{\omega}$ versus the driving amplitude $\xi$ for various dc bias current amplitudes $\alpha$ and $\gamma=10, \omega \tau=5$. The results display strong nonlinearity for small bias and ohmic-resistancelike behavior for high bias. Again, little difference is apparent between the classical and quantum corrected curves for high driving amplitudes $\xi$, demonstrating that quantum effects are most pronounced in the linear region (small $\xi$ ) being negligible for large $\xi$, showing once again how nonlinear effects mask the quantum effects for large $\xi$. This is corroborated in Fig. 6, where again the quantum effects are most obvious in the linear-response region $\xi=0.01$. On increasing $\xi$, that is, proceeding from the linear region to the nonlinear region, one also perceives masking by the nonlinearity, which is evident in Fig. 7.

To conclude, we have demonstrated how the QSE may be used to calculate quantum corrections to the nonlinear impedance and current-voltage characteristics of the Josephson tunneling junction in the zero-capacitance limit, by evaluating the ac nonlinear response in the presence of noise for wide ranges of the noise strength $\gamma$, the dc bias current $\alpha$, and the nonlinearity $\xi$. In order to obtain these results, we have solved the QSE using matrix-continued fractions, allowing us to determine various characteristics of the noninertial Brownian motion in a tilted cosine potential in the presence of a large ac driving force. We finally remark that the treatment outlined here may be extended to the general inertial (or nonzero-capacitance) case. The zero-capacitance (noninertial) limit used in the present paper automatically

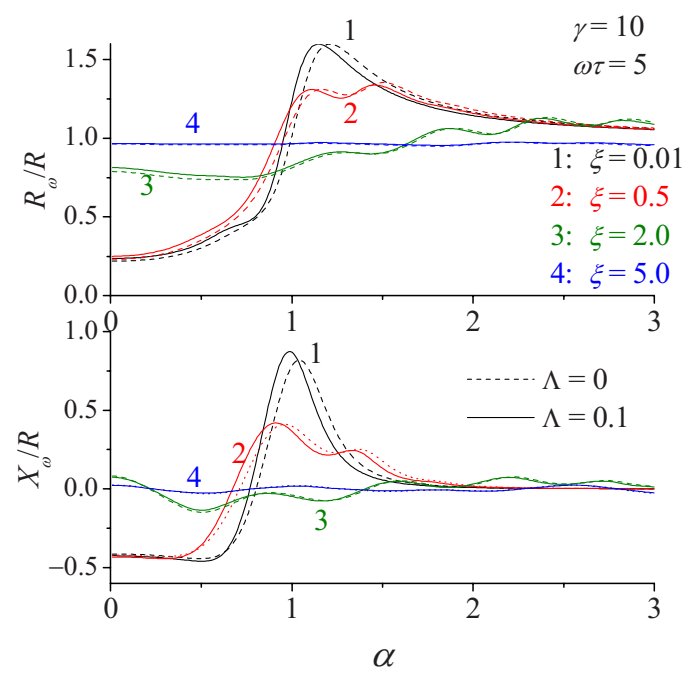

FIG. 6. (Color online) Normalized dynamic resistance $R_{\omega}$ and reactance $X_{\omega}$ vs the dc bias amplitude $\alpha$ for various ac amplitudes $\xi$ and $\gamma=10, \omega \tau=5$, showing relatively strong quantum effects for small stimuli (curve 1) indicating that the quantum behavior depends more strongly on the dc bias for small ac amplitudes than relatively large ones (curves 3 and 4). Dashed and solid lines are the classical and quantum results, respectively.

restricts the band of frequencies in which the model is applicable to frequencies much less than the Josephson plasma frequency $\omega_{p}=\sqrt{2 I e / \hbar C} .{ }^{19-21}$ If one wishes to treat accurately the $\mathrm{GHz}$ and $\mathrm{THz}$ regions, the complete phase-space distribution $W(\varphi, p, t) \quad(p=m \dot{\varphi})$ must be used, giving rise, ${ }^{15-18,30}$ on expansion of the momentum part of the distribution in orthogonal Hermite polynomials, to a hierarchy of partial differential recurrence relations in configuration space. The actual configuration-space distribution $P(\varphi, t)$ must then be extracted from the hierarchy, usually by continued fractions, with the much simpler QSE naturally emerging $^{15,17}$ from the hierarchy in the high-damping limit

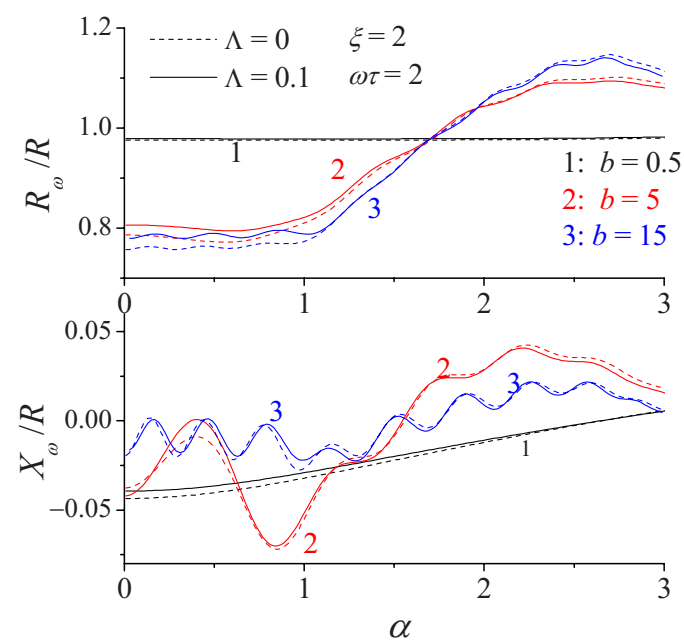

FIG. 7. (Color online) Normalized dynamic resistance $R_{\omega}$ and reactance $X_{\omega}$ vs the dc bias amplitude $\alpha$ for various Josephson coupling energies $\gamma$ and $\xi=2, \omega \tau=2$, showing that the quantum effects are relatively small compared to the nonlinear ones. Dashed and solid lines are the classical and quantum results, respectively. 
as originally demonstrated by Brinkman for the classical case. $^{54}$

\section{ACKNOWLEDGMENTS}

IRCSET, The Trinity College Dublin Trust, and The Trinity College Visiting Lecturers Fund are thanked for financial support for this work.

\section{APPENDIX A: DERIVATION OF EQ. (11) DIRECTLY FROM THE QUANTUM LANGEVIN EQUATION}

By way of illustration of the Langevin equation method of generating the hierarchy of differential recurrence relations for the statistical moments, we shall demonstrate how averaging of the semiclassical noninertial Langevin equation corresponding to the QSE (3) over its realizations yields the same hierarchy of equations as the QSE. The Langevin equation corresponding to Eq. (3) in the Stratonovich interpretation $^{55}$ for the random variable $\varphi(t)$ reads

$$
\dot{\varphi}(t)=-\frac{1}{\zeta} \partial_{\varphi}\left\{V[\varphi(t)]+\frac{\zeta}{2} D[\varphi(t)]\right\}+\sqrt{\frac{\beta}{\zeta} D[\varphi(t)]} L(t),
$$

where the dot denotes the time derivative and $L(t)$ is a random current with Gaussian white-noise properties, viz.,

$$
\overline{L(t)}=0, \quad \overline{L(t) L\left(t^{\prime}\right)}=2 k T R^{-1} \delta\left(t-t^{\prime}\right) .
$$

(The overbar means the statistical average over the realizations of the random current). However, one must remember that the quantum Langevin equation is written down from $a$ priori knowledge of the QSE, which is dissimilar to the situation encountered in the classical case. There the Langevin equation is written down independently of either the FokkerPlanck or SE, and the results of the two methods only coincide as a consequence of the Gaussian white-noise properties of $L(t)$, particularly Isserlis's theorem (Wick's theorem) ${ }^{30}$ is satisfied. This theorem allows multiple time correlations of Gaussian random variables to be expressed as two time correlations. Now $\varphi(t)$ is governed by the Langevin Eq. (A1), which contains a multiplicative noise term and is of the standard classical form ${ }^{18}$

$$
\dot{\varphi}(t)=h[\varphi(t)]+g[\varphi(t)] L(t) .
$$

The corresponding Langevin equation for the evolution of an arbitrary function $f[\varphi(t)]$ whose expectation value we wish to calculate is then

$$
\dot{f}[\varphi(t)]=h[\varphi(t)] \frac{d}{d \varphi} f[\varphi(t)]+g[\varphi(t)] \frac{d}{d \varphi} f[\varphi(t)] L(t) .
$$

(Here we have noted that in the transformation of variables in a Stratonovich stochastic differential equation, one can apply the usual rules of calculus. ${ }^{18,30}$ ) Next, we recall the theorem ${ }^{18,30}$ that the averaged evolution equation for a function $f[\varphi(t)]$ is given by

$$
\begin{aligned}
\frac{d}{d t}\langle f(\varphi)\rangle= & \left\langle h(\varphi) \frac{d}{d \varphi} f(\varphi)\right\rangle \\
& +\frac{2 k T}{R}\left\langle g(\varphi) \frac{d}{d \varphi}\left[g(\varphi) \frac{d}{d \varphi} f(\varphi)\right]\right\rangle,
\end{aligned}
$$

hence, with

$$
f[\varphi(t)]=e^{-i n \varphi(t)},
$$

Eq. (A4) with the potential Eq. (7) yields the recurrence Eq. (11) for $c_{n}(t)=\left\langle e^{-i n \varphi(t)}\right\rangle$.

\section{APPENDIX B: MATRIX-CONTINUED FRACTION SOLUTION OF EQ. (13)}

The dual-index scalar five-term recurrence relation, Eq. (13), can be rewritten in terms of solvable matrix three-term recurrence relations as

$$
\mathbf{Q}_{1}^{-} \mathbf{C}_{0}+\mathbf{Q}_{1} \mathbf{C}_{1}+\mathbf{Q}_{1}^{+} \mathbf{C}_{2}=-\mathbf{F} \mathbf{C}_{1}^{*}
$$

and

$$
\mathbf{Q}_{n}^{-} \mathbf{C}_{n-1}+\mathbf{Q}_{n} \mathbf{C}_{n}+\mathbf{Q}_{n}^{+} \mathbf{C}_{n+1}=0
$$

Here the column vectors $\mathbf{C}_{ \pm n}$ are

$$
\begin{aligned}
& \mathbf{C}_{0}=\left(\mathbf{c}_{0}\right), \quad \mathbf{C}_{ \pm n}=\left(\begin{array}{c}
\mathbf{c}_{ \pm 2 n} \\
\mathbf{c}_{ \pm(2 n-1)}
\end{array}\right), \quad n= \pm 1, \pm 2, \ldots, \\
& \mathbf{c}_{0}=\left(\begin{array}{c}
\vdots \\
0 \\
1 \\
0 \\
\vdots
\end{array}\right), \quad \mathbf{c}_{n}=\left(\begin{array}{c}
\vdots \\
c_{n}^{-1}(\omega) \\
c_{n}^{0}(\omega) \\
c_{n}^{1}(\omega) \\
\vdots
\end{array}\right),
\end{aligned}
$$

the supermatrices $\mathbf{Q}_{n}, \mathbf{Q}_{n}^{ \pm}$, and $\mathbf{F}$ are given by

$$
\begin{gathered}
\mathbf{Q}_{n}=\left(\begin{array}{cc}
\mathbf{q}_{2 n} & \mathbf{q}_{2 n}^{-} \\
\mathbf{q}_{2 n-1}^{+} & \mathbf{q}_{2 n-1}
\end{array}\right), \quad \mathbf{Q}_{n}^{+}=\left(\begin{array}{cc}
\overline{\mathbf{q}}_{2 n} \mathbf{i} & \mathbf{q}_{2 n}^{+} \\
0 & \overline{\mathbf{q}}_{2 n-1} \mathbf{i}
\end{array}\right), \\
\mathbf{Q}_{n}^{-}=\left(\begin{array}{cc}
\bar{q}_{2 n} \mathbf{i} & 0 \\
\mathbf{q}_{2 n-1}^{-} & \bar{q}_{2 n-1} \mathbf{i}
\end{array}\right)
\end{gathered}
$$

with

$$
\mathbf{Q}_{1}^{-}=\left(\begin{array}{c}
\bar{q}_{2} \mathbf{i} \\
\mathbf{q}_{1}^{-}
\end{array}\right) \text {and } \mathbf{F}=\left(\begin{array}{cc}
0 & 0 \\
0 & \bar{q}_{1} \mathbf{f}
\end{array}\right),
$$

where $\mathbf{i}$ denotes the unit matrix, and $\mathbf{f}$ is given by

$$
\mathbf{f}=\left(\begin{array}{ccccc}
\ddots & \vdots & \vdots & \vdots & . \\
\cdots & 0 & 0 & 1 & \cdots \\
\cdots & 0 & 1 & 0 & \cdots \\
\cdots & 1 & 0 & 0 & \cdots \\
. & \vdots & \vdots & \vdots & \ddots
\end{array}\right),
$$




$$
\begin{gathered}
\mathbf{q}_{n}=i\left(\begin{array}{ccccc}
\ddots & \vdots & \vdots & \vdots & . \\
\cdots & z_{n}^{-1}(\omega) & \xi & 0 & \cdots \\
\cdots & \xi & z_{n}^{0}(\omega) & \xi & \cdots \\
\cdots & 0 & \xi & z_{n}^{1}(\omega) & \cdots \\
. & \vdots & \vdots & \vdots & \ddots
\end{array}\right), \\
\mathbf{q}_{n}^{ \pm}=\left(\begin{array}{ccccc}
\ddots & \vdots & \vdots & \vdots & . \\
\cdots & q_{n}^{ \pm} & \pm 3 i \xi \bar{q}_{n} / 4 & 0 & \cdots \\
\cdots & \pm 3 i \xi \bar{q}_{n} / 4 & q_{n}^{ \pm} & \pm 3 i \xi \bar{q}_{n} / 4 & \cdots \\
\cdots & 0 & \pm 3 i \xi \bar{q}_{n} / 4 & q_{n}^{ \pm} & \cdots \\
. & \vdots & \vdots & \vdots & \ddots
\end{array}\right) .
\end{gathered}
$$

Thus, the column vectors $\mathbf{C}_{n}$ can be calculated from Eqs. (B1) and (B2) via matrix continued fractions as the matrix products $^{26}$

$$
\begin{aligned}
& \mathbf{C}_{n}=\mathbf{S}_{n} \mathbf{S}_{n-1} \ldots \mathbf{S}_{2} \mathbf{C}_{1}, \\
& \mathbf{C}_{1}=\mathbf{S}_{1} \mathbf{C}_{0}+\Delta_{1} \mathbf{F C}_{1}^{*},
\end{aligned}
$$

where $\mathbf{S}_{n}$ and $\boldsymbol{\Delta}_{1}$ are matrix continued fractions defined by the recurrence equation

$$
\begin{gathered}
\mathbf{S}_{n}=\left(-\mathbf{Q}_{n}-\mathbf{Q}_{n}^{+} \mathbf{S}_{n+1}\right)^{-1} \mathbf{Q}_{n}^{-}, \\
\boldsymbol{\Delta}_{n}=\left(-\mathbf{Q}_{n}-\mathbf{Q}_{n}^{+} \boldsymbol{\Delta}_{n+1} \mathbf{Q}_{n+1}^{-}\right)^{-1} .
\end{gathered}
$$

Next we introduce complex vectors and matrices via

$$
\mathbf{C}_{1}=\mathbf{C}_{1}^{\prime}+i \mathbf{C}_{1}^{\prime \prime}, \quad \mathbf{S}_{1}=\mathbf{S}_{1}^{\prime}+i \mathbf{S}_{1}^{\prime \prime}, \quad \overline{\mathbf{F}}=\boldsymbol{\Delta}_{1} \mathbf{F}=\overline{\mathbf{F}}^{\prime}+i \overline{\mathbf{F}}^{\prime \prime}
$$

so that we have from Eq. (B4)

$$
\begin{aligned}
& \left(\mathbf{I}-\overline{\mathbf{F}}^{\prime}\right) \mathbf{C}_{1}^{\prime}-\overline{\mathbf{F}}^{\prime \prime} \mathbf{C}_{1}^{\prime \prime}=\mathbf{S}_{1}^{\prime} \mathbf{C}_{0}, \\
& \left(\mathbf{I}+\overline{\mathbf{F}}^{\prime}\right) \mathbf{C}_{1}^{\prime \prime}-\overline{\mathbf{F}}^{\prime \prime} \mathbf{C}_{1}^{\prime}=\mathbf{S}_{1}^{\prime \prime} \mathbf{C}_{0},
\end{aligned}
$$

where I denotes the unit matrix. Ultimately, we have for the real and imaginary parts in the simultaneous matrix Eqs. (B5) and (B6)

$$
\begin{aligned}
& \mathbf{C}_{1}^{\prime}=\left[\mathbf{I}-\overline{\mathbf{F}}^{\prime}-\overline{\mathbf{F}}^{\prime \prime}\left(\mathbf{I}+\overline{\mathbf{F}}^{\prime}\right)^{-1} \overline{\mathbf{F}}^{\prime \prime}\right]^{-1}\left[\mathbf{S}_{1}^{\prime}+\overline{\mathbf{F}}^{\prime \prime}\left(\mathbf{I}+\overline{\mathbf{F}}^{\prime}\right)^{-1} \mathbf{S}_{1}^{\prime \prime}\right] \mathbf{C}_{0}, \\
& \mathbf{C}_{1}^{\prime \prime}=\left[\mathbf{I}+\overline{\mathbf{F}}^{\prime}-\overline{\mathbf{F}}^{\prime \prime}\left(\mathbf{I}-\overline{\mathbf{F}}^{\prime}\right)^{-1} \overline{\mathbf{F}}^{\prime \prime}\right]^{-1}\left[\mathbf{S}_{1}^{\prime \prime}+\overline{\mathbf{F}}^{\prime \prime}\left(\mathbf{I}-\overline{\mathbf{F}}^{\prime}\right)^{-1} \mathbf{S}_{1}^{\prime}\right] \mathbf{C}_{0} .
\end{aligned}
$$

${ }^{1}$ E. P. Wigner, Phys. Rev. 40, 749 (1932).

${ }^{2}$ J. E. Moyal, Proc. Camb. Philos. Soc. 45, 99 (1949).

${ }^{3}$ S. R. de Groot and L. G. Suttorp, Foundations of Electrodynamics (North-Holland, Amsterdam, 1972).

${ }^{4}$ M. Hillery, R. F. O'Connell, M. O. Scully, and E. P. Wigner, Phys. Rep. 106, 121 (1984).

${ }^{5}$ H. W. Lee, Phys. Rep. 259, 147 (1995).

${ }^{6}$ W. P. Schleich, Quantum Optics in Phase Space (Wiley-VCH, Berlin, 2001).

${ }^{7}$ Quantum Mechanics in Phase Space, edited by C. K. Zachos, D. B. Fairlie, and T. L. Curtright (World Scientific, Singapore, 2005).

${ }^{8}$ R. E. Wyatt, Quantum Dynamics with Trajectories: Introduction to Quantum Hydrodynamics (Springer, New York, 2005).

${ }^{9}$ U. Weiss, Quantum Dissipative Systems, 3rd ed. (World Scientific, Singapore, 2008).

${ }^{10}$ G. S. Agarwal, Phys. Rev. A 4, 739 (1971).

${ }^{11}$ A. O. Caldeira and A. J. Leggett, Physica A 121, 587 (1983).

${ }^{12}$ Y. Tanimura and P. G. Wolynes, Phys. Rev. A 43, 4131 (1991); J. Chem. Phys. 96, 8485 (1992).

${ }^{13}$ D. Kohen, C. C. Marston, and D. J. Tannor, J. Chem. Phys. 107, 5236 (1997); D. Kohen and D. J. Tannor, Adv. Chem. Phys. 111, 219 (2000).

${ }^{14}$ J. L. García-Palacios, Europhys. Lett. 65, 735 (2004); J. L. García-Palacios and D. Zueco, J. Phys. A 37, 10735 (2004).

${ }^{15}$ W. T. Coffey, Yu. P. Kalmykov, S. V. Titov, and B. P. Mulligan, Phys. Chem. Chem. Phys. 9, 3361 (2007); Europhys. Lett. 77, 20011 (2007); Phys. Rev. E 75, 041117 (2007).

${ }^{16}$ W. T. Coffey, Yu. P. Kalmykov, and S. V. Titov, J. Chem. Phys. 127, 074502 (2007).

${ }^{17}$ W. T. Coffey, Yu. P. Kalmykov, S. V. Titov, and B. P. Mulligan,
J. Phys. A: Math. Theor. 40, F91 (2007); 40, 12505 (2007).

${ }^{18} \mathrm{H}$. Risken, The Fokker-Planck Equation, 2nd ed. (SpringerVerlag, Berlin, 1989).

${ }^{19} \mathrm{G}$. Barone and A. Paterno, Physics and Applications of the Josephson Effect (Wiley, New York, 1982).

${ }^{20}$ K. K. Likharev, Dynamics of Josephson Junctions and Circuits (Gordon and Breach, New York, 1986).

${ }^{21}$ M. Tinkham, Introduction to Superconductivity, 2nd ed. (McGraw-Hill, New York, 1996).

${ }^{22}$ P. Fulde, L. Pietronero, W. R. Schneider, and S. Strässler, Phys. Rev. Lett. 35, 1776 (1975); W. Dieterich, P. Fulde, and I. Peschel, Adv. Phys. 29, 527 (1980).

${ }^{23}$ J. D. Cresser, D. Hammonds, W. H. Louisell, P. Meystre, and H. Risken, Phys. Rev. A 25, 2226 (1982); W. W. Chow, J. GeaBanacloche, L. M. Pedrotti, V. Sanders, W. Schleich, and M. O. Scully, Rev. Mod. Phys. 57, 61 (1985); W. T. Coffey, Yu. P. Kalmykov, and E. S. Massawe, Phys. Rev. E 48, 699 (1993).

${ }^{24}$ A. J. Viterbi, Principles of Coherent Communication (McGrawHill, New York, 1966).

${ }^{25}$ G. Grüner, A. Zawadowski, and P. M. Chaikin, Phys. Rev. Lett. 46, 511 (1981); S. G. Chung, Phys. Rev. B 29, 6977 (1984).

${ }^{26}$ M. Evstigneev, O. Zvyagolskaya, S. Bleil, R. Eichhorn, C. Bechinger, and P. Reimann, Phys. Rev. E 77, 041107 (2008).

${ }^{27}$ V. A. Shklovskij and O. V. Dobrovolskiy, Phys. Rev. B 78, 104526 (2008).

${ }^{28}$ V. Ambegaokar and B. I. Halperin, Phys. Rev. Lett. 22, 1364 (1969).

${ }^{29}$ Yu. M. Ivanchenko and L. A. Zil'berman, Zh. Eksp. Teor. Fiz. 55, 2395 (1968) [Sov. Phys. JETP 28, 1272 (1969).

${ }^{30}$ W. T. Coffey, Yu. P. Kalmykov, and J. T. Waldron, The Langevin Equation, 2nd ed. (World Scientific, Singapore, 2004), Chap. 5. 
${ }^{31}$ W. T. Coffey, Yu. P. Kalmykov, S. V. Titov, and L. Cleary, Phys. Rev. E 78, 031114 (2008).

${ }^{32}$ E. P. Wigner, Z. Phys. Chem. Abt. B 19, 203 (1932).

${ }^{33}$ S. Shapiro, Phys. Rev. Lett. 11, 80 (1963).

${ }^{34}$ H. Kanter and F. L. Vernon, Jr., J. Appl. Phys. 43, 3174 (1972).

${ }^{35}$ J. McDonald and J. R. Clem, Phys. Rev. B 56, 14723 (1997).

${ }^{36}$ Z. Zhai, P. V. Parimi, and S. Sridhar, Phys. Rev. B 59, 9573 (1999).

${ }^{37}$ Z. Zhai, H. Srikanth, S. Sridhar, A. Erb, E. Walker, and R. Flukinger, Physica C 282-287, 1601 (1997).

${ }^{38}$ P. P. Nguyen, D. E. Oates, G. Dresselhaus, and M. S. Dresselhaus, Phys. Rev. B 48, 6400 (1993).

${ }^{39}$ B. B. Jin and R. X. Wu, J. Appl. Phys. 84, 3250 (1998).

${ }^{40}$ Y. M. Habib, C. J. Lehner, D. E. Oates, L. R. Vale, R. H. Ono, G. Dresselhaus, and M. S. Dresselhaus, Phys. Rev. B 57, 13833 (1998).

${ }^{41}$ D. E. Oates, P. P. Nguyen, Y. M. Habib, G. Dresselhaus, M. S. Dresselhaus, G. Koren, and E. Polturak, Appl. Phys. Lett. 68, 705 (1996).

${ }^{42}$ L. M. Xie, J. Wosik, and J. C. Wolfe, Phys. Rev. B 54, 15494 (1996).

${ }^{43}$ W. T. Coffey, J. L. Déjardin, and Yu. P. Kalmykov, Phys. Rev. E 61, 4599 (2000).

${ }^{44}$ W. T. Coffey, J. L. Déjardin, and Yu. P. Kalmykov, Phys. Rev. B 62, 3480 (2000).
${ }^{45}$ Single Charge Tunneling, edited by H. Grabert and M. H. Devoret (Plenum Press, New York, 1992).

${ }^{46}$ M. H. Devoret, D. Esteve, C. Urbina, J. Martinis, A. Cleland, and J. Clarke, in Quantum Tunneling in Solids, edited by Yu. Kagan and A. J. Leggett (Elsevier, Amsterdam, 1992).

${ }^{47}$ G. L. Ingold and H. Grabert, Phys. Rev. Lett. 83, 3721 (1999); H. Grabert, G. L. Ingold, and B. Paul, Europhys. Lett. 44, 360 (1998).

${ }^{48}$ L. Machura, M. Kostur, P. Talkner, J. Łuczka, and P. Hänggi, Phys. Rev. E 73, 031105 (2006).

${ }^{49}$ J. Ankerhold, Quantum Tunnelling in Complex Systems (Springer-Verlag, Berlin, 2007).

${ }^{50}$ J. T. Anderson and A. M. Goldman, Phys. Rev. Lett. 23, 128 (1969).

${ }^{51}$ M. Simmonds and W. H. Parker, Phys. Rev. Lett. 24, 876 (1970).

${ }^{52}$ C. M. Falco, W. H. Parker, S. E. Trullinger, and P. K. Hansma, Phys. Rev. B 10, 1865 (1974).

${ }^{53}$ P. Joyez, D. Vion, M. Gotz, M. H. Devoret, and D. Esteve, J. Supercond. 12, 757 (1999).

${ }^{54}$ H. C. Brinkman, Physica 22, 29 (1956).

${ }^{55}$ R. L. Stratonovich, Conditional Markov Processes and Their Application to the Theory of Optimal Control (Elsevier, New York, 1968). 